\title{
Investigation of thermodynamic properties of single-layer two-component coatings
}

\author{
Stanislav Vlasov ${ }^{1}$, Aleksey Chikhranov ${ }^{2,}{ }^{*}$, Aleksandr Averyanov ${ }^{1}$, Valentina Vlasova $^{3}$, and \\ Yana Dolzhenko ${ }^{2}$ \\ ${ }^{1}$ Federal State Autonomous Educational Institution of Higher Education National Research Nuclear \\ University MEPhI (Moscow Engineering Physics Institute), Moscow, 115409, Russian Federation \\ ${ }^{2}$ Federal State Budgetary Educational Institution of Higher Education Ulyanovsk Institute of Civil \\ Aviation named after Chief Marshal of Aviation B.P. Bugaev, Ulyanovsk, Russian Federation \\ ${ }^{3}$ Federal State Budgetary Educational Institution of Higher Education KG Razumovsky Moscow State \\ University of Technologies and Management (FCU), Moscow, 109004, Russian Federation
}

\begin{abstract}
The results of studying the dynamics of disordered alloys by the method of collective variables are presented. The densities of phonon states of disordered alloys with BCC structure of the kxrb1-x system are calculated. The contribution of phonons to the free energy and entropy of the alloy at different temperatures is determined. It is shown that the contribution of phonons to the total free energy of the alloy decreases with decreasing temperature. The contribution of phonons to the entropy of alloys at $300 \mathrm{~K}$ is crucial.
\end{abstract}

\section{Introduction}

Recently, there has been a sharp increase in interest in studying the dynamics of disordered systems, the simplest example of which is binary substitution alloys. Two directions can be distinguished in the theory of dynamic properties of refractory alloys. One of them is associated with the use of various modifications of the theory of a self-consistent field, and the second - with numerical methods. Each approach has its own advantages and limitations. For example, the coherent potential method does not take into account the effects of fluctuations in the local environment in the vicinity of the substitution atoms of the matrix crystal. The introduction of local corrections (clusters, etc.) leads to excessive complications of the method. Implementation of machine experiment methods is associated with great computational difficulties.

In this paper, the lattice dynamics of disordered alloys is studied by the method of collective variables. In this case, within the framework of a unified approach, it is possible to consistently take into account the effects of correlations and ordering.

\footnotetext{
* Corresponding author: chihranov@mail.ru
} 


\section{Formulation of the problem}

The Hamiltonian of a binary alloy in the approximation of paired interionic interactions can be written as follows [1]:

$$
\begin{gathered}
H=\frac{1}{2} \sum_{R_{i}}\left[M_{1}\left(\delta R_{i}\right)^{2} \frac{1+\sigma_{1}}{2}\right]+\frac{1}{2 N} \sum_{R_{i}, R_{J}}\left\{\sum _ { q } \left[V_{11}(q) \frac{1+\sigma_{j}}{2} \frac{1+\sigma_{1}}{2}+2 V_{12}(q) \frac{1+\sigma_{1}}{2} \frac{1-\sigma_{j}}{2}+\right.\right. \\
\left.\left.V_{22}(q) \frac{1-\sigma_{j}}{2} \frac{1-\sigma_{1}}{2}\right] e^{i q\left(R_{I}-R_{J}\right)}\right\} .
\end{gathered}
$$

The first term in (1) is the kinetic energy, and the second is the potential energy of the alloy. The following notation is introduced: $M_{\chi}$ - is the mass of an ion of the $\chi$ type $(\chi=1$, 2); $\delta R_{i}$ - the displacement vector of the ion located at the site $R_{i}$ from the equilibrium position $R_{i}^{0} ; V_{\chi \chi^{\prime}}(q)$ - is the Fourier transform of the effective pair interaction of ions of grades $\chi, \chi^{\prime}$. Explicit expressions for $V_{\chi \chi}(q)$ are given in [1]. The occupation number operator $\sigma_{j}$-takes values 1 or -1 , depending on whether the site $R_{i}$ is occupied by an ion of type 1 or $2 ; N-$ is the number of lattice sites. To simplify the entries, (1) presents the Hamiltonian of a substitution alloy whose unit cell contains one atom (alloys of BCC and FCC structures).

We restrict ourselves to the harmonic approximation and turn to general variables [2].

\section{Numerical results}

In this paper the results of the theory are illustrated by calculating the density of disordered alloys with a bcc structure of the system $\mathrm{K}_{x} \mathrm{Rb}_{1-x}$. The concentration $\mathrm{x}$ varied in the entire range of values $0 \leq x \leq 1$. It was of interest to clarify the relative contribution of atomic vibrations to the thermodynamic properties of alloys. Alloys of the K-Rb system in the rigid lattice approximation were studied by us in [1,3]. This work is a natural continuation of the works [3, 4].

As a first step, the calculation of the density of phonon states was performed in Matlab. The calculations were carried out for three temperatures: 300, 200, $100 \mathrm{~K}$. At these temperatures, the system forms a continuous series of solid solutions. A model pseudopotential [10] was used in calculating the Fourier transforms of the effective interionic potentials $V_{\chi \chi}(q)\left(\chi, \chi^{\prime}=\mathrm{K}, \mathrm{Rb}\right)$.

When the alloy concentration and temperature change, the shape of the dispersion curves of the middle crystal does not change. Only the values of the frequencies $\bar{\omega} k \lambda$ change, passing at $\mathrm{x}=0$ to the spectrum of pure rubidium, and at $\mathrm{x}=1$ - to pure potassium. Based on the curves obtained for $\bar{\omega} k \lambda$ the density of phonon statesg $(\omega)$ was calculated. The values of $g(\omega)$ are normalized to unity:

$$
\int_{0}^{\omega_{\max }} g(\omega) d \omega=1,
$$

where $\omega_{\max }-$ maximum frequency.

In PVCs, the frequencies $\omega k \lambda$, and hence $g(\omega)$, depend on temperature indirectly, via the equilibrium atomic volume. If the density of phonon states is known, then it is easy to calculate the contribution of atomic vibrations to various thermodynamic functions.

The contribution of phonons to the free energy $F$ and the entropy of the alloy $S$ at 100 , 200 , and $300 \mathrm{~K}$ was studied. The calculation of $F_{p h}, S_{p h}$ was carried out by the formulas (per atom).

$$
F_{P H}=3 k_{B} T \int_{0}^{\omega_{\max }} \ln \left[2 \operatorname{sh}\left(\frac{h \omega}{2 k_{B} T}\right)\right] g(\omega) d \omega
$$




$$
s_{p h}=3 k_{B} \int_{0}^{\omega_{\max }}\left\{\frac{h \omega}{2 k_{B} T} \operatorname{cth}\left(\frac{h \omega}{2 k_{\mathrm{B}} T}\right)-\operatorname{In}\left(\frac{h \omega}{2 k_{\mathrm{B}} T}\right)\right\} g(\omega) d \omega,
$$

where $k_{B}$ - is the Boltzmann constant. The expression for the free energy $F_{0}$ (the alloy atoms are in the equilibrium position) is given in $[3,4]$. The table shows the results of calculations. It is seen that the contribution of phonons to the total free energy of the alloy $F=F_{0}+F_{p h}$ decreases with decreasing temperature, as it should be. The relative share of $F_{p h}$ in $F$ is about $2-3 \%$. The contribution of phonons to the entropy of alloys at $300 \mathrm{~K}$ is decisive. In the region of low concentrations $(x \leq 0,1)$ and at other temperatures, the configurational component of the entropy $S_{\text {conf }}$ is significantly less than $S_{p h}$. The role of configuration entropy increases with decreasing temperature. For example, at $100 \mathrm{~K} S_{p h}$ and $S_{\text {conf }}$ are comparable in magnitude (see table 1).

In the virtual crystal approximation, the search for the poles of the Green's function $G$ is reduced to solving the following eigenvalue problem:

$$
\widetilde{W} \tilde{a}=0,
$$

where $\widetilde{W}-$ is a $3 \times 3$ matrix; $\tilde{\mathrm{a}}-$ is a column vector, the elements of which are $a_{k}(a-x, y$, $z) ; x, y, z$-components of the unit polarization vector; $a_{k}$ - amplitudes of the displacement vector $\delta R$. The matrix elements of $\widetilde{W}$ are equal to

$$
\begin{gathered}
\overline{w_{\alpha \beta}}(k)=\bar{M} \omega_{k}^{2} \delta_{\alpha \beta}-\int_{Q}\left[\left(Q_{\alpha}+\kappa_{\alpha}\right)\left(Q_{\beta}+\kappa_{\beta}\right) \bar{V}(Q+\kappa)+\left(Q_{\alpha}-\kappa_{\alpha}\right)\left(Q_{\beta}-\right.\right. \\
\left.\left.-\kappa_{\beta}\right) \bar{V}(Q-\kappa)-2 Q_{\alpha} Q_{\beta} \bar{V}(Q)\right] d Q
\end{gathered}
$$

The formula above contains the notation

$$
\bar{V}(q)=c_{1}^{2} V_{11}(q)+2 c_{1} c_{2} V_{12}(q), \quad \bar{M}=c_{1} M_{1}+c_{2} M_{2}
$$

Table 1. Contribution of atomic vibrations to the free energy and entropy of the system's alloys $\mathrm{K}_{x} \mathrm{Rb}_{1-x}$

\begin{tabular}{|c|c|c|c|c|c|c|c|}
\hline \multirow{2}{*}{$T, \mathrm{~K}$} & $x$ & $\begin{array}{c}F, \\
\mathrm{Ry} / \text { ion }\end{array}$ & $\begin{array}{c}\mathrm{F}_{\mathrm{ph}} \\
\mathrm{Ry} / \text { ion }\end{array}$ & $\frac{F_{p h}}{F} 100 \%$ & $\begin{array}{c}\mathrm{S} \cdot 10^{4} \\
\mathrm{Ry} /(\text { ion } \cdot \mathrm{K})\end{array}$ & $\begin{array}{c}\mathrm{S}_{\mathrm{ph}} \cdot 10^{4} \\
\mathrm{Ry} /(\mathrm{ion} \cdot \mathrm{K})\end{array}$ & $\frac{S_{p h}}{S} 100 \%$ \\
\hline \multirow{5}{*}{300} & 0 & -0.3790 & -0.0108 & 2.85 & 0.5499 & 0.5499 & 100 \\
\cline { 2 - 8 } & 0.2 & -0.3861 & -0.0104 & 2.69 & 0.6075 & 0.5362 & 88.3 \\
\cline { 2 - 8 } & 0.5 & -0.3934 & -0.0097 & 2.47 & 0.6231 & 0.5129 & 82.3 \\
\cline { 2 - 8 } & 0.8 & -0.3968 & -0.0088 & 2.22 & 0.5584 & 0.4841 & 86.7 \\
\cline { 2 - 8 } & 1.0 & -0.3970 & -0.0081 & 2.04 & 0.4604 & 0.4604 & 100 \\
\hline \multirow{5}{*}{200} & 0 & $-0,3739$ & $-0,0056$ & 1,50 & 0,4711 & 0,4711 & 100 \\
\cline { 2 - 8 } & 0.2 & $-0,3809$ & $-0,0053$ & 1,39 & 0.5507 & 0,4572 & 83,0 \\
\cline { 2 - 8 } & 0,5 & $-0,3884$ & -0.0048 & 1,24 & 0,5835 & 0,4336 & 74,3 \\
\cline { 2 - 8 } & 0,8 & $-0,3921$ & $-0,0043$ & 1,10 & 0,5035 & 0,4049 & 80,4 \\
\cline { 2 - 8 } & 1,0 & $-0,3722$ & $-0,0038$ & 1,02 & 0,3813 & 0,3813 & 100 \\
\hline \multirow{3}{*}{100} & 0 & -0.3770 & -0.0014 & 0.37 & 0.3385 & 0.3385 & 100 \\
\cline { 2 - 8 } & 0.2 & -0.3769 & -0.0013 & 0.34 & 0.4947 & 0.3250 & 65.7 \\
\cline { 2 - 8 } & 0.5 & -0.3852 & -0.0011 & 0.29 & 0.6067 & 0.3018 & 49.7 \\
\cline { 2 - 8 } & 0.8 & -0.3886 & -0.0008 & 0.21 & 0.4566 & 0.2738 & 60.0 \\
\cline { 2 - 7 } & 1.0 & -0.3895 & -0.0005 & 0.13 & 0.2513 & 0.2513 & 100 \\
\hline
\end{tabular}

Explicit analytical expressions for $V_{\chi \chi} \cdot(q),\left(\chi, \chi^{\prime}=1,2\right)$ are given in [4].

The study of the role of fluctuation effects in the dynamics of disordered systems will be the subject of the next article. 


\section{References}

1. S. Vlasov, A. Averyanov, V. Tabakov, V. Vlasova, MATEC Web Conf., 298, (2019)

2. A. Averyanov, E. Rotanov, S. Vlasov, E3S Web Conf., 126, (2019)

3. G.C. Nina, A.F. Ogori, M. Ukeyima el at., The Journal of Microbiology, Biotechnology and Food Sciences, 9, (2019)

4. S. Vlasov, V. Vlasova, A. Zentsov, Springer International Publishing AG, 5, (2020). 